\title{
SARS-CoV-2 vaccines: anamnestic response in previously infected recipients
}

\author{
Jyothi N. Purushotham ${ }^{1,2}$, Neeltje van Doremalen ${ }^{1}$ and Vincent J. Munster (iD)
}

Cell Research (2021) 31:827-828; https://doi.org/10.1038/s41422-021-00516-7

\begin{abstract}
The continued evolution of SARS-CoV-2 has raised questions regarding the ability of prior immunity to early pandemic strains to afford protection against emerging variants. In a recent study, Stamatatos et al. demonstrate that currently approved mRNA vaccines elicit antibodies capable of neutralizing heterologous antigen and they further show that single-dose vaccination triggers an anamnestic response in individuals with pre-existing anti-RBD IgG developed through previous SARS-CoV-2 infection.
\end{abstract}

The mRNA vaccines produced by Moderna (mRNA-1273) and Pfizer/BioNTech (BNT162b2) comprise an essential part of the global vaccination campaign to combat COVID-19. These vaccines encode stab25ilized forms of the SARS-CoV-2 spike glycoprotein, promoting the induction of robust titers of antibodies with potent virus neutralizing function. Like other currently available vaccines, these immunogens were designed based on the original WuhanHu-1 virus strain. The recent emergence of SARS-CoV-2 variants, potentially exhibiting increased transmissibility and/or pathogenicity, pose a threat to pandemic control efforts. ${ }^{1}$ Of major public health concern is whether or not immunity to early pandemic strains, developed via vaccination or previous infection, will still confer protection against newly circulating variants.

The B.1.351 lineage, first detected in South Africa, has raised particular alarm due to reductions in efficacy observed for vaccines tested in regional clinical trials, such as ChAdOx $1 \mathrm{nCoV}$ 19 (AZD1222) developed by AstraZeneca/Oxford. ${ }^{2}$ In a recent report in Science, Stamatatos et al. investigate neutralizing antibody responses against B.1.351 strains in the sera of naïve and previously infected recipients of BNT162b2 or mRNA-1273. ${ }^{3}$ The B.1.351 lineage encompasses the following spike protein amino acid substitutions: K417N, E484K, and N501Y in the receptor-binding domain (RBD); D614G and frequently A701V in S2; D80A, D215G, and occasionally a 242-244 deletion in the Nterminal domain (NTD). ${ }^{1,4}$ In their experiments, the authors assess the susceptibility to neutralization of a pseudovirus expressing B.1.351 spike in comparison to a vaccine-matched Wuhan-Hu-1 pseudovirus.

Functional studies have demonstrated the reduced sensitivity of variants to early pandemic monoclonal antibodies, convalescent plasma, and vaccine-induced neutralizing antibodies. ${ }^{4}$ Similarly, Stamatatos et al. show that although two doses of mRNA vaccine elicited neutralizing antibodies against Wuhan-Hu-1 in previously naïve individuals, titers against B.1.351 were significantly depressed. In individuals with prior SARS-CoV-2 infection, relatively weak titers of neutralizing antibodies against Wuhan-Hu-1 and low or undetectable titers against B.1.351 were measured pre vaccination. However, in those that had developed RBD-specific lgG ${ }^{+}$memory $B$ cell and antibody responses during infection, a single immunization triggered an anamnestic response that boosted not only the autologous neutralizing titers (by 1000-fold), but also heterologous titers against B.1.351, albeit to a lesser extent (3-fold lower). Analogous results were reported in a recent study by Goel et al., demonstrating the amplification of neutralizing titers against D614G and B.1.351 pseudoviruses in individuals with prior infection after a single dose of mRNA vaccine, with little improvement observed after dose two. ${ }^{5}$ Notably, Stamatatos et al. also show that B.1.351 neutralizing titers in previously infected vaccinees after a single dose were comparable to or higher than autologous neutralizing titers in previously naïve vaccinees after two doses of mRNA vaccine. ${ }^{3}$ This result is significant given that the latter is associated with greater than $90 \%$ protective efficacy against COVID19 in clinical trials. 6,7

These data are supported by research suggesting that repeated or prolonged exposure to antigen promotes the diversification of the neutralizing antibody response and targeting of variable epitopes. The development of cross-reactive antibody responses has been explored for other highly mutable pathogens, such as HIV, influenza A virus, and hepatitis $C$ virus. Studies from these fields indicate that the breadth of neutralization is contingent upon antigen persistence, which fosters the progressive accumulation of somatic mutations and affinity maturation. ${ }^{8}$ Accruement of a heterogenous repertoire of memory $B$ cells may promote the ability of antibody-producing cells to recognize and respond to related but distinct targets. Collectively, this literature points to time and exposure to antigenic variants as key determinants of resilience to viral mutation. Consistent with this hypothesis, Muecksch et al. recently characterized clonally related antibodies isolated from a cohort of individuals approximately one and six months post SARS-CoV-2 infection. Somatic mutations acquired after several months of convalescence bestowed certain RBDspecific antibodies with greater neutralization potency and more restricted options for viral escape, in comparison to those recovered shortly after infection. ${ }^{9}$ Vaccination did not increase levels of somatic hypermutation in memory B cell clones isolated from previously infected individuals in the study performed by Goel et al. However, analyses spanned only several weeks after vaccination, which may have been premature to observe substantial evolution in the $B$ cell repertoire. ${ }^{5}$

\footnotetext{
${ }^{1}$ Laboratory of Virology, Rocky Mountain Laboratories, National Institute of Allergy and Infectious Diseases, National Institutes of Health, Hamilton, MT, USA and ${ }^{2}$ The Jenner Institute, Nuffield Department of Clinical Medicine, University of Oxford, Oxford, UK

Correspondence: Vincent J. Munster (vincent.munster@nih.gov)
}

Published online: 18 May 2021 
Overall, this evidence provides support for the continuation of current COVID-19 vaccination campaigns despite the emergence of new viral variants. The findings in previously infected vaccinees suggest that when variant-specific vaccines become available, a synergistic boosting effect may be expected, given that prior vaccine recipients should have pre-existing anti-RBD lgG and/or memory $B$ cells. Moreover, a recent study by Moyo-Gwete et al. demonstrated that B.1.351 infection elicits antibodies with crossvariant neutralizing potential, suggesting that second-generation vaccines, featuring the B.1.351 spike mutations, may be especially effective in stimulating a broadly neutralizing response. ${ }^{10}$ Findings from both Stamatatos et al. and Muecksch et al. suggest that an interval of at least several months, between delivery of firstgeneration and new variant-specific vaccines, may be optimal for affinity maturation and the diversification of antibody-producing cells. Furthermore, only a single dose may be required to efficiently boost heterologous responses to levels associated with significant protection against COVID-19. In summary, the investigation performed by Stamatatos et al. may inform strategies to optimize protective immunity in the context of continued SARSCoV-2 evolution.

\section{ADDITIONAL INFORMATION}

Competing interests: The authors declare no competing interests.

\section{REFERENCES}

1. Tegally, H. et al. Nature 592, 438-443 (2021).

2. Madhi, S. A. et al. N. Engl. J. Med. https://doi.org/10.1056/NEJMoa2102214 (2021).

3. Stamatatos, L. et al. Science https://doi.org/10.1126/science.abg9175 (2021).

4. Zhou, D. et al. Cell 184, 2348-2361 (2021).

5. Goel, R. R. et al. Sci. Immunol. 6, eabi6950 (2021).

6. Baden, L. R. et al. N. Engl. J. Med. 384, 403-416 (2020).

7. Polack, F. P. et al. N. Engl. J. Med. 383, 2603-2615 (2020).

8. Sprenger, K. G. et al. Proc. Natl. Acad. Sci. USA 117, 20077-20087 (2020).

9. Muecksch, F. et al. bioRxiv https://doi.org/10.1101/2021.03.07.434227 (2021).

10. Moyo-Gwete, T. et al. N. Engl. J. Med. https://doi.org/10.1056/NEJMc2104192 (2021). 